\title{
O sistema celulolítico de microrganismos anaeróbios: uma mini-revisão sobre o celulossoma
}

\author{
Eduardo de A. Ximenes ${ }^{1}$ \\ Carlos R. Felix ${ }^{2}$
}

\begin{abstract}
O termo biomassa, de acordo com Linko e cols. (19), pode ser usado para qualquer planta ou outro material formado em grandes quantidades. Sua efetiva utilização em processos biológicos pode ser um meio importante para contornar a escassez de comida, rações e combustíveis que poderá acontecer em um futuro próximo, levando em consideração o crescente aumento populacional humano. Considerando ainda que os principais componentes da biomassa são polímeros consistindo de glicose e outros açúcares relacionados, estes se tornarão as principais fontes de carbono e energia para processos de fermentação microbiana, depois de serem convertidos em monossacarídeos. Por outro lado, como a biomassa é em grande parte originária de tecido vegetal rígido, consistindo principalmente de celulose, hemicelulose, lignina e proteínas, métodos efetivos de solubilização e hidrólise devem ser desenvolvidos para a efetiva utilização destes compostos. Para o propósito de aumentar a solubilização de material vegetal fibroso, celulases e enzimas relacionadas, apresentando alta atividade enzimática, e os respectivos genes têm sido investigados há bastante tempo (24). Dentre as celulases, destacam-se o sistema celulolítico produzido pelos microrganismos anaeróbios, ou seja, o celulossoma, com sua exuberante estrutura molecular.
\end{abstract}

\section{O sistema celulolítico das bactérias anaeróbias}

As bactérias anaeróbias tendem a produzir complexos multi-enzimáticos chamados de celulossoma, os quais estão ligados à superfície celular e consistem de enzimas conhecidas como exoglucanases, endoglucanases, xilanases,

\footnotetext{
${ }^{1}$ Centro Universitário de Brasília.Brasília-DF-Brasil-Endereço eletrônico: eduardoax@ucsal.br

${ }^{2}$ Laboratório de Enzimologia- Departamento de Biologia Celular-Universidade de Brasília, Brasília-DF-

Brasil.CEP.70910900 Endereço eletrônico: carlosrf@unb.br
} 
mananase, liquenase, esterase feruloil, além de proteínas com função estrutural $(3,13,23)$. Como exemplo podemos citar o celulossoma produzido pela bactéria Clostridium thermocellum $(10,27)$.

A estrutura do celulossoma é altamente ordenada. Já se sabe há algum tempo que a alta atividade deste no processo de hidrólise de celulose cristalina é governada por sua estrutura quaternária única $(10,27)$.

O celulossoma de $C$. thermocellum é composto por em torno de 26 subunidades diferentes $(8,13,14)$. Sua massa molecular tem sido estimada estar na faixa de 2 × $10^{6}$ a $6.5 \times 10^{6} \mathrm{Da}(8,13,28)$. Ele também pode se agrupar formando uma estrutura maior chamada de policelulossoma, com uma massa molecular variando de $50 \times 10^{6}$ a $80 \times 10^{6} \mathrm{Da}(8)$.

O celulossoma tem uma estrutura muito rígida, que não é facilmente rompida por agentes desnaturantes. O tratamento com sódio dodecil sulfato (SDS) na presença de EDTA e tióis parece ser o mais eficiente caminho para dissociá-lo (28). Esta dificuldade de dissociação tem dificultado o isolamento de suas subunidades e o estudo de sua estrutura quaternária. É importante mencionar que o tratamento com SDS conduz à quase completa perda da capacidade de hidrolisar celulose cristalina. Contudo, muito pouca ou nenhuma perda de atividade de endoglucanase é observada (8).

Cada uma das subunidades do celulossoma possui um domínio não catalítico (domínio de peptídeo repetido-DPR ou domínio “dockerin"), que é conservado, interagindo com uma proteína estrutural (conhecida como Cip A, com massa molecular de $250 \mathrm{kDa}$ ), não catalítica, contendo um módulo de ligação a carboidrato (MLC) e 9 domínios repetidos, conhecidos como elementos internos repetidos ou coesinas $(3,13,14)$. A interação é dependente da presença de cálcio (Fig.1) (8, 21).

Quatro genes de espécies de Clostridium codificando proteínas estruturais têm sido sequenciados. Todos eles contêm domínios múltiplos, chamados de "coesina", capazes de se ligarem aos DPR mencionados no parágrafo anterior. Um centro altamente hidrofóbico provavelmente contribui para a alta estabilidade dos domínios de coesina, os quais resistem a tratamentos com proteases e detergentes. A proteína estrutural, conforme mencionado anteriormente, contém um MLC que é responsável pela ligação do complexo ao substrato celulose $(3,13,14)$. 
CB

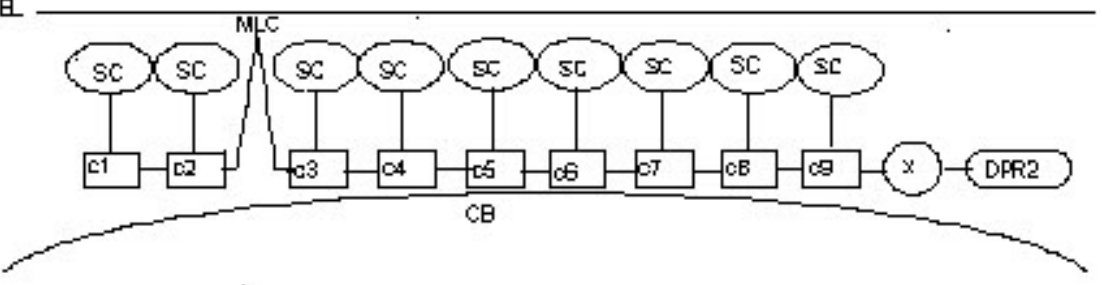

Figura 1 - Representação esquemática do celulossoma de Clostridium thermocellum. Cel= celulose; $c 1-9=$ coesinas; $M L C=$ módulo de ligação a carboidrato; $S C=$ diferentes subunidades catalíticas; $X=$ módulo hidrofílico; $D P R 2=$ domínio de peptídeo repetido 2; $C B=$ célula bacteriana. A interação entre as subunidades catalíticas e as coesinas se dá na presença de cálcio.

Outro domínio, chamado de DPR do tipo 2, tem sido encontrado em proteína estrutural de $C$. thermocellum. Este domínio está envolvido na ligação da proteína estrutural à parede celular da bactéria, onde ela interage com um outro tipo de coesina, chamada do tipo 2 , presente em diferentes proteínas da superfície celular. É importante salientar que tanto o DPR quanto a coesina dos tipos 2 diferem consideravelmente em termos de homologia dos respectivos tipos 1. Por outro lado, alguns resíduos estruturais importantes são conservados no caso dos diferentes tipos de coesina, sugerindo que eles compartilham uma mesma estrutura tridimensional $(3,13,14)$.

O fato de que peptídeo sinal tem sido encontrado em todos os polipeptídeos codificados pelos genes estruturais dos componentes celulossomais (o que sugere que eles são secretados individualmente pela via de secreção comum) e a proteína estrutural não ter sido encontrada como um polipeptídeo individual no meio de cultura, dá suporte a idéia de que o celulossoma de $C$. thermocellum é montado e organizado na superfície das células $(10,21,27)$.

\section{Fungos anaeróbios}

Fungos anaeróbios têm sido isolados do trato alimentar de herbívoros e outros ambientes (4). De acordo com padrões morfológicos, estes fungos têm sido divididos em dois grupos : monocêntricos (contém um esporângio) e policêntrico (contém múltiplos esporângios) (5). O primeiro grupo inclui espécies de Neocallimastix (25), Caecomyces (29) e Piromyces (1) ; o segundo grupo contém 
espécies de Orpinomyces (1), Anaeromyces (7) e Ruminomyces (12). A classificação destes microrganismos foi motivo de controvérsia por vários anos, e muito ainda se discute hoje em termos de classificação ao nível de espécie $(6,11,15,22)$. Estes fungos degradam rapidamente material de origem vegetal (20) e possuem uma organela, chamada hidrogenossoma, que está envolvida na geração de energia (30).

Fungos anaeróbios produzem diferentes enzimas com altas atividades no processo enzimático de degradação da parede celular de planta, incluindo celulases, ??glucanases, ?-glucosidases, xilanases, mananases, acetil-xilana esterases e esterases fenólicas $(4,17)$. Diferentes enzimas têm sido clonadas e sequenciadas dos monocêntricos Neocallimastix patriciarum (2,9) e Piromyces sp (26) e também do fungo policêntrico Orpinomyces sp cepa PC-2 (20). Algumas das enzimas são encontradas livres, enquanto outras fazem parte de um complexo multi-proteico de alta massa molecular similar ao celulossoma de Clostridia $(16,26)$. Contudo, quando comparado às bactérias anaeróbias, os estudos sobre o complexo proteico de alta massa molecular produzidos pelos fungos anaeróbios, apesar da grande quantidade de dados gerada nos últimos anos, encontram-se em um estágio praticamente inicial e muito ainda precisa ser feito para um melhor entendimento do mesmo.

\section{Considerações finais}

Bactérias e fungos anaeróbios produzem um complexo multi-proteico de alta-massa molecular (celulossoma), otimizado no sentido de apresentar um sinergismo intramolecular pela interação de todos os componentes em uma ordem e taxa efetiva. Este sistema altamente organizado confere aos celulossomas a capacidade de hidrolisar material de origem vegetal de forma extremamente eficiente, o que possibilita o uso do mesmo em várias aplicações biotecnológicas. Estudos recentes visando à produção de celulossomas quiméricos, com subunidades catalíticas escolhidas especificamente para uma determinada aplicação biotecnológica, têm sido realizados. Apesar de toda a pesquisa desenvolvida nesta área até o momento, um processo comercialmente efetivo ainda está distante. Mais estudos precisam ser realizados no sentido de se compreender melhor o mecanismo de ação celulossomal, assim como ainda se faz necessária a busca de novas cepas capazes de produzir enzimas individuais ou complexos proteicos com alta atividade específica. 


\section{Referências}

1. Barr, D. J. S., H. Kudo, K. D. Jakober \& K. -J. Cheng. (1989). Morphology and development of rumen fungi: Neocallimastix sp, Piromyces communis and Orpinomyces bovis gen. nov., sp nov. Can. J. Bot. 67: 2815-2824.

2. Black., G. M., G. P. Hazlewood, G. P. Xue, C. G. Orpin \& H. J. Gilbert. (1994). Xylanase B from Neocallimastix patriciarum contains a non-catalytic 455-residue linker sequence comprised of 57 repeats of an octapeptide. Biochem. J. 299: 381387.

3. Blum, D. L., I .A. Kataeva, X.-L. Li \& L. G. Ljungdahl. (2000). Feruloyl esterase activity of the Clostridium thermocellum cellulosome can be attributed to previously unknown domains of Xyn Y and Xyn Z. J. Bacteriol. 182 (5): 1346-1351.

4. Borneman, W. S., D. E. Akin \& L. G. Ljungdahl. (1989). Fermentation products and plant cell wall-degrading enzymes produced by monocentric and polycentric anaerobic ruminal fungi. Apple. Environ. Microbiol. 55: 1066-1073.

5. Borneman, W. S. \& D. E. Akin. (1994). The nature of the anaerobic fungi and their polysaccharide degrading enzymes. Mycoscience 35: 199-211.

6. Braune, R. (1913). Untersuchungen über die im Wiederkauermagen vorkommeneden Protozoen. Arch. Protistenkd. 32: 11-170.

7. Breton, A., A. Bernalier, M. Dusser, G. Fonty, B. Gallard-Martinie \& J. Guillot. (1990). Anaeromyces mucronatus nov.gen., nov. sp. A new strictly anaerobic rumen fungus with polycentric thallus. FEMS Microbiol. Lett. 70: 177-182.

8. Choi, S. K. \& L. G. Ljungdahl. (1996). Dissociation of the cellulosome of Clostridium thermocellum in the presence of ethylenediaminetetraacetic acid occurs with the formation of truncated polypeptides. Biochemistry 35: 4897-4905.

9. Denman, S., G. P. Xue \& B. Patel. (1996). Characterization of a Neocallimastix patriciarum cellulase cDNA (celA) homologous to Trichoderma reesei cellobiohydrolase II. Appl. Environ. Microbiol. 297: 359-364.

10. Felix, C. R. \& L. G. Ljungdahl. (1993). The cellulosome : the exocellular organelle in Clostridium. Annu. Rev. Microbiol. 47: 791-819.

11. Heath, I. B. \& T. Bauchop. (1985). Mitosis and the phylogeny of the genus Neocallimastix. Can. J. Bot. 63: 159-1604.

12. Ho, Y. W. \& T. Bauchop. (1990). Ruminomyces elegans gen.et sp nov. A polycentric anaerobic rumen fungus from cattle. Mycotaxon 38:397-405.

13. Kataeva, I., X.-L. Li, H. Chen, S.-K. Choi \& L. G. Ljungdahl. (1999). Cloning and sequence analysis of a new cellulase gene encoding CelK, a major cellulosome component of Clostridium thermocellum: Evidence for gene duplication and Recombination. J. Bacteriol. 181 (17): 5288-5295.

14. Kataeva, I.A., R. D. Seidel III, A. Shah, L. T. West, X.-L Li \& L. G. Ljungdahl. (2002). The fibronectin type 3-like repeat from the Clostridium thermocellum 
cellobiohydrolase CbhA promotes hydrolysis of cellulose by modifying its surface. Appl. Environ. Microbiol. 68(9): 4292-4300.

15. Li., J. \& B. Heath. (1992). The phylogenetic relationships of the anaerobic chytridiomycetous gut fungi (Neocallimasticaceae) and the Chytridiomycota.I. Cladistic analysis of rRNA sequences. Can. J. Bot. 70: 1738- 1746.

16. Li X.-L., H. Z. Chen, \& L. G. Ljungdahl. (1997). Monocentric and polycentric anaerobic fungi produce structurally related cellulases and xylanases. Appl. Environ. Microbiol. 63: 628-635.

17. Li X.-L., H. Z. Chen \& L. G. Ljungdahl. (1997). Two cellulases, CelA and CelC, from the polycentric fungus Orpinomyces sp. strain PC-2 contain N-terminal docking domains for a cellulose-hemicellulose complex. Appl. Environ. Microbiol. 63: 4721-4728.

18. Liebetanz, E. (1910). Die parasitischen Protozoen des Wiederkauermagens. Arch. Protistenkd. 19: 19-80.

19. Linko, M., K. Pountanen \& L. Viikari. (1989). In : Coughlan, M. P. (Ed), Enzyme Systems for Lignocellulose Degradation, Elsevier Applied Science, London, pp 331.

20. Ljungdahl, L. G., D. L. Blum, H. Z. Chen, Y. He, I. Kataeva, X. -L. Li, E. A. Ximenes. (1998). The cellulase/hemicellulose system of the anaerobic fungus Orpinomyces and aspects of further cellulase research. In: Genetics, Biochemistry and Ecology of Cellulose Degradation, Ohmyia, K., K. Hayashi, K. Sakka, Y. Kobayashi, S. Sarita, and T. Kimiura (Eds), Mie Bioforum 98, Uni Plubishers Co., Ltd, Tokyo, Japan, pp 495-506.

21. Lytle, B., W. M. Westler \& J. H. D. Wu. (1998). Molecular assembly of the Clostridium thermocellum cellulosome. In: Genetics, Biochemistry and Ecology of Cellulose Degradation, Ohmyia, K., K. Hayashi, K. Sakka, Y. Kobayashi, S. Sarita, and T. Kimiura (Eds), Mie Bioforum 98, Uni Plubishers Co., Ltd, Tokyo, Japan, pp 449-449.

22. Munn, E. A., C. A. Greenwood \& C. G. Orpin. (1987). Organization of the kinetosomes and associated structures of zoospores of the rumen chytridiomycete Neocallimastix patriciarum. Can. J. Bot. 65: 456-465.

23. Ohmiya K., T. Fujino, J. Sukhumavasi \& S. Shimizu. (1989). Cloning of an endo1,4-?-D-glucanase gene from Clostridium josui and its expression in Escherichia coli. Appl. Environ. Microbiol. 55: 2399-2402.

24. Ohmiya, K., K. Sakka, S. Karita \& T. Kimura. (1997). Structure of cellulases and their application. Biotechnol. Gen. Engineer. Rev. 14: 365-414.

25. Orpin, C. G. (1975). Studies on the rumen flagellate Neocallimastix frontalis. J.Gen. Microbiol. 91: 249-262.

26. Steenbakkers, P. J. M., X.L. Li., E.A. Ximenes, J. G. Arts, H. Chen, L. G Ljungdahl \& H. J. M. Op Den Camp. (2001). Noncatalytic Docking domains of cellulossomes of anaerobic fungi. J. Bacteriol. 183 (18): 5325-5333. 
27. Shoham, Y., R. Lamed and E. A. Bayer. (1999). The cellulosome concept as an efficient microbial strategy for the degradation of insoluble polysaccharides. Trends in Microbiology. 275 (7): 275-280.

28. Wu, J. H. D., W. H. Orme-Johnson \& A. L. Demain. (1988). Two components of an extracellular protein aggregate of Clostridium thermocellum together degrade crystalline cellulose. Biochemistry 27: 1703-1709.

29. Wubah, D. A. \& M. S. Fuller. (1991). Studies on Caecomyces communis. Morphology and development. Mycologia 83: 303-310.

30. Yarlett, N., C. G. Orpin, E. A. Munn, N. C. Yarlett \& C. A. Greenwood. (1986). Hydrogenosomes in the rumen fungus Neocallimastix. Biochem. J. 236: 729-739. 